\title{
Using Social Media to Geo-Target Emergency Management Efforts
}

\author{
Bandana Kar* \\ karb@ornl.gov \\ Oak Ridge National Laboratory \\ Oak Ridge, TN, USA \\ Nathaniel Dede-Namfo \\ nd1115@txstate.edu \\ Texas State University \\ San Marcos, TX, USA
}

\author{
Edwin Chow \\ chow@txstate.edu \\ Texas State University \\ San Marcos, TX, USA \\ Xiaohui Liu \\ xiaohuiliugis@gmail.com \\ Dalhousie University \\ Halifax, Nova Scotia, Canada
}

\begin{abstract}
The ubiquity of social media data has increased their use during emergency management (EM) in near real-time. For instance, during Haiti Earthquake (2010) and Hurricane Harvey (2017), Ushahidi and Twitter were used respectively for emergency response and rescue operations. Nonetheless, social media data tend to contain [ir]relevant information to be useful for disaster analytics for EM efforts. In this study, geo-tagged tweets obtained for 2013 Colorado flooding were analyzed to determine (i) what kind of situational awareness (SA) information could be extracted from tweets for emergency response and (ii) what is the spatio-temporal distribution of such information. The results indicate that tweets generated before September $12^{\text {th }}$ (day of heavy precipitation) were non-relevant, but tweets generated on and following September $12^{\text {th }}$ contained crisis information (about the event and its impacts), warnings, preparatory information. Next phase of this study will focus on developing a framework to integrate SA information with physical risk and social vulnerability to geo-target EM efforts.
\end{abstract}

\section{CCS CONCEPTS}

- Information systems $\rightarrow$ Content analysis and feature selection; Data analytics.

\section{KEYWORDS}

situational awareness, crisis informatics, emergency management, natural language processing

\section{ACM Reference Format:}

Bandana Kar, Edwin Chow, Nathaniel Dede-Namfo, and Xiaohui Liu. 2019. Using Social Media to Geo-Target Emergency Management Efforts. In 5th ACM SIGSPATIAL International Workshop Emergency Management Using GIS (EMGIS'19), November 5, 2019, Chicago, IL, USA. ACM, New York, NY, USA, 4 pages. https://doi.org/10.1145/3356998.3365769

*All authors contributed equally to this research.

ACM acknowledges that this contribution was authored or co-authored by an employee, contractor, or affiliate of the United States government. As such, the United States government retains a nonexclusive, royalty-free right to publish or reproduce this article, or to allow others to do so, for government purposes only.

EMGIS'19, November 5, 2019, Chicago, IL, USA

(C) 2019 Association for Computing Machinery.

ACM ISBN 978-1-4503-6965-7/19/11 ..\$15.00

https://doi.org/10.1145/3356998.3365769

\section{INTRODUCTION}

Gaining insight into what happened before, during and after a disaster event is essential for emergency response and recovery efforts. Traditionally, socio-economic and geospatial data sets available from census and other conventional sources are used for these efforts. These data sets, however, (i) are not available in real-time, and (ii) do not capture a community's response and recovery needs. By contrast, social media data (e.g., tweets) are available in near real-time and contain information about users' feelings and needs.

Among the social media platforms, Twitter has become popular for emergency management (EM) activities as it is free, instantaneous, globally distributed, and provides contextual information [3]. Twitter earthquake detector developed by USGS is a very good example of using Twitter for disseminating risk information about earthquakes within minutes of receiving information from seismometers and tweets [1]. Twitter has also been used for coordinating relief efforts, raising awareness and helping with emergency preparedness $[8,16,18]$. Several studies have also used tweets to extract information about damages sustained and level of threat based on users' posting habits, tweet volume and sentiments [5, 7].

Despite the increase in tweet characters from 140 to 280, the information disseminated by a single tweet can be confusing and may not contain relevant and reliable information to be used by emergency managers. Information available from tweets also tend to vary across disaster events $[4,10]$. Tweets also tend to be noisy and biased based on users' demography, and may contain [ir] relevant information for disaster analytics [4,9].Currently, several tools and data sets containing crisis-related terms (e.g. CrisisLex, EMT Terms, VerbNet) are available to classify tweets into various categories for use during EM activities [11, 12, 15]. However, these tools generally do not classify tweets into categories representative of EM phases to provide situational awareness (SA) information, which is crucial for EM efforts [15]. This study focused on analyzing geo-tagged tweets which were obtained during 2013 Colorado flooding to determine (i) the types of SA information that could be extracted from tweets before, during and after the flooding event, and (ii) explore the spatio-temporal distribution of SA information.

The remainder of the paper is organized into following sections. A brief review of the existing techniques and tools to classify tweets related to disasters is presented in section 2. The data and methodology used to answer the research questions are discussed in section 3. Results and a discussion of the findings are presented in section 
4, which is followed by concluding remarks and a discussion of future research directions in section 5 .

\section{JUSTIFICATION OF WORK}

Kongthon et al. [6] investigated the different type of SA information that were available from tweets during 2011 flood in Thailand. The authors found an overall increase in tweet volume before the flood event, and an increase in number of retweets for certain users who provided locational and situational information about the flood event. Their analyses also revealed that most tweets provided upto-date information about the flood, road conditions, and contained requests for relief efforts. In a similar study, Shaw et al.[13] developed a typology of Twitter usage during 2010 Queensland flood in Australia. Takahashi, Tandoc and Carmichael [14] examined Twitter usage during and after Typhoon Haiyan that impacted Philippines in 2013 based on time and geographic location of users and their extent of social media engagement. The authors found that the Twitter use varied among users, and stakeholders used Twitter to disseminate different information. For instance, the public used Twitter to coordinate relief efforts, journalists used Twitter for sharing information. Wang and Zhuang [17] analyzed tweets generated during 2012 Hurricane Sandy by governmental organizations (GO), non-governmental organizations (NGO) and news agents using five key performance indicators - impression, like, mention, response time and retweet count. The authors found that news agents generated a large volume of tweets, but the retweet count was higher for tweets generated by GO and NGOs.

Studies have also used tweets from multiple disaster events to (i) develop frameworks for classifying tweets accurately into hazard and EM related categories, such as death, injury, warnings, damages, etc., and (ii) develop a database of EM terms to aid with tweet classification for emergency response efforts. Zhang and Vucetic [19] used a semi-supervised approach (crowdsourcing to manually classify tweets and Logistic Regression to classify tweets based on their scoring) to classify tweets obtained during six disasters which occurred between October 2012 and July 2013: hurricane Sandy, Boston Bombings, Oklahoma Tornado, West Texas Explosion, Alberta Floods, Queensland Floods. By using unlabelled tweets with a trained classifier, the authors found that the choice of algorithm and corpus of tweets impacted classification accuracy. Imran and Castillo [2] used human experts and Latent Dirichlet Allocation method to classify tweets obtained during several disasters, which included wild fire, flood, typhoon and earthquake from different countries to develop a framework to classify tweets into information categories, such as hazard type, damages, sympathy/opinion, etc. Temnikova, Castillo and Vieweg [15] developed a database known as EMTerms that contains terminologies related to hazard events and emergency management to aid with tweet classification. The authors classified 500 tweets each from four different events, which included 2013 Russia-China floods, 2013 Pakistan-Afghanistan floods, 2013 Bohol earthquake and 2012 Colorado wildfires, manually and using Conditional Random Fields method (a probabilistic method used in Natural Language Processing) to develop a sequence of terms for different EM related topics. The authors developed a trained model using the classified data set, which they used subsequently on 35 anthropogenic and natural hazard events to develop the final EMTerms list for classification of tweets generated during other disasters. Olteanu et al. [11] used tweets from six different disasters (included hurricane, bombing, tornado, flood and explosion) that occurred in the USA, Canada and Australia to create CrisisLex-a dictionary of crisis related terms similar to EMTerms.

Currently, CrisisLex contains 380 terms and EMTerms contains 23 categories and 7,200 terms that could be used to classify tweets related to disasters (CrisisLex 2018). Given the number of terms available from CrisisLex and EMTerms, it is not always possible to use these dictionaries to classify tweets into useful categories. Furthermore, Twitter use varies across different phases of a disaster, is dependent on the type, location and time of the event, and is related to users' demography. It is therefore essential to extract specific SA information from tweets to geo-target emergency response efforts. This study, therefore, focused on developing a framework to classify tweets before, during and after an event into specific SA categories to help with EM efforts, and associate the SA information with physical risk and social vulnerability of impacted counties to geo-target response activities. The framework can be used to undertake strategic crowdsourcing activities to help with EM efforts by emergency management agencies and first responders.

\section{DATA AND METHODS}

For this study, 1,195,183 firehose tweets were obtained from Twitter Inc. during September 9th to 17th for the 2013 Colorado flood. Among the tweets, $85 \%$ were in English, and 1.38\% were geo-tagged. From the 15,154 geotagged tweets, 4,829 tweets posted by 2,039 unique users located within Colorado were extracted. For each tweet, special characters and encoding, such as \#, @, RT, etc. were removed, and apostrophes (e.g. I'm) were replaced with a stroke (i.e. $I \backslash \mathrm{m}$ ) to differentiate text from the string wrapper.

Half of the geo-tagged tweets (training data set) were manually classified into four broad categories - Information, Conversation, Others and Not Relevant and thirteen classes - (Information category was classified into: Preparation, Sharing, Warning, Loss, Location; Conversation tweets were classified into: Positive, Negative, Humour, Concerns, Neutral; tweets belonging to Others class were categorized into: Advertisement, Help; Non-relevant tweets were not categorized). Each word in a tweet was tokenized and converted into a string vector to examine the relationship between its occurrence and a specific category/class. A few machine-learning algorithms, such as J48, Naïve Bayes Multinomial (NBM), Sequential Minimal Optimization (SMO) and Random Forest (RF) were evaluated based on percentage of correct prediction, kappa statistics, confusion metric and areas under the Receiver Operating Characteristic (ROC) Curve. Based on the percentage of correct prediction, kappa statistics, confusion metric and areas under the ROC Curve, the results of SMO were used to classify the tweets. Finally, the spatio-temporal distribution of tweet classes were analyzed for each day during September $9^{\text {th }}$ thorugh September $17^{\text {th }}$ to understand which contextual tweets were predominant before, during and after the flood event. The spatial distribution of tweets on the day of heavy precipitation was analyzed with regard to the extent of damage, extent of precipitation and population density. 


\section{RESULTS AND DISCUSSION}

Of the geocoded tweets, $35 \%$ was non-relevant, $42 \%$ contained floodrelated information, $22 \%$ was conversations about the event, and $1 \%$ was classified as "Others". From the informational tweets, about $54 \%$ was categorized as "sharing" that provided news/updates about the event, $22 \%$ contained warning messages, $16 \%$ reported selfidentified location, $5 \%$ reported damages/losses, and the remaining $3 \%$ provided advice for disaster preparation. Among the $16 \%$ locational tweets, some provided contextual information (e.g. who they were with) or reported building/institution names. About $52 \%$ of the "conversation" tweets contained neutral discussion about the event. The remaining "conversation" tweets expressed positive emotions (11\%), negative emotions (13\%), concerns (12\%) and humor (12\%). Of the tweets categorized as "Others", $46 \%$ contained disaster-related advertisements (e.g. plumbing, locksmith) and 54\% contained solicitations about assistance (figure 1).

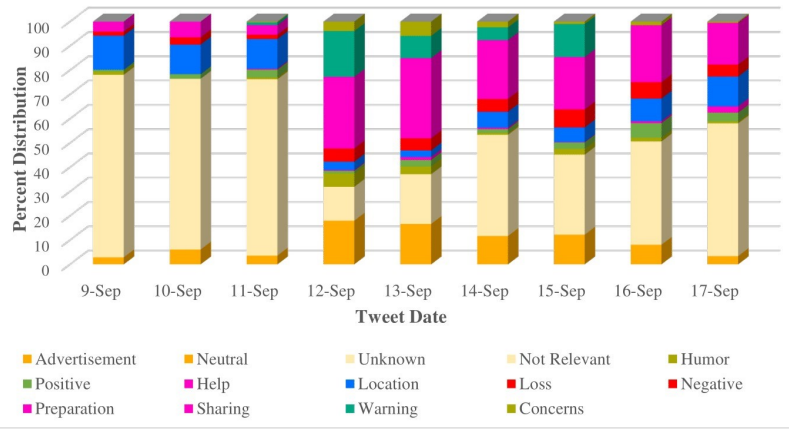

Figure 1: Temporal Distribution of Tweets

About $30-70 \%$ of the tweets generated before and after September $12^{\text {th }}$ and $13^{\text {th }}$ (the days when heavy precipitation occurred) were non-relevant. About $86 \%$ of the tweets generated on Sep. $12^{\text {th }}$ was relevant, which contained neutral discussions (18\%), news/updates about the event (28\%), warnings and alert messages (19\%), concerns (4\%), humor (6\%), location information (3.5\%), positive and negative emotions (4.5\%), and damages and preparatory information (3\%). A similar trend was observed on Sep. $13^{\text {th }}$ with $79 \%$ of the tweets being relevant, which contained neutral discussions (16\%), sharing (31\%), warnings $(9 \%)$, concerns $(6 \%)$, humor (3\%), location information $(2.7 \%)$, positive and negative emotions $(6.5 \%)$, and damages, preparatory information and solicitation for help (4.8\%). The sptial distribution of tweets (Figure 2) also indicates that the tweets that were generated within the worst damaged counties of Larimer, Boulder and Clear Creek contained information about loss and damages, locations of potential shelters, and shared information about the event. These tweets also contained more concerns and negative opinions. The tweets that were generated in the moderately damaged counties of Denver, Gilpin and Broomfield contained humor, neutral and negative sentiments.

About 70 to $80 \%$ of the geo-tagged tweets were clustered in the moderately to worst damaged counties of Morgan, Logan, Boulder, Larimer, Clear Creek, Jefferson, Adams, Arapahoe, El Paso, and Fremont. The tweets that provided advertisements, preparatory information, loss and damage information were concentrated in

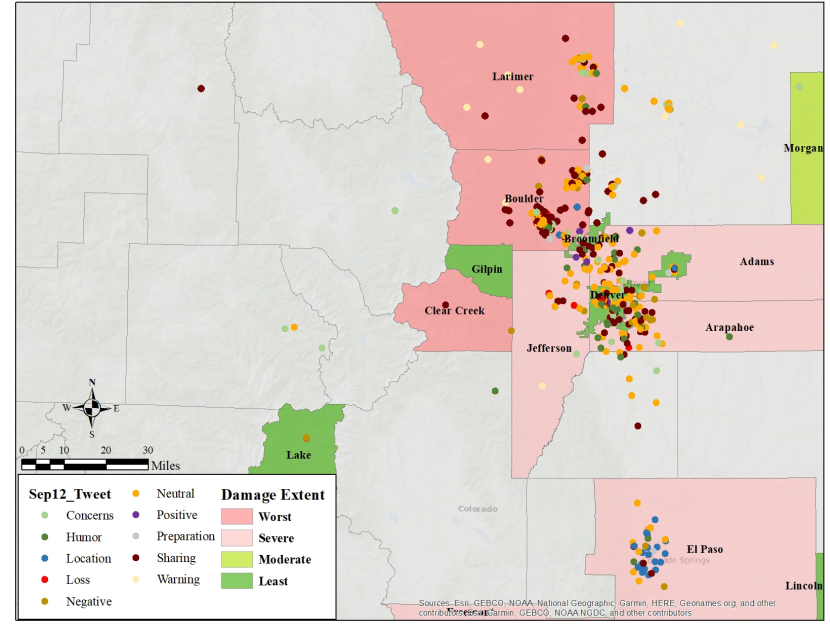

Figure 2: Spatial Distribution of Tweets on Sept. $12^{\text {th }}, 2013$

Denver and Broomfield cities, worst damaged counties of Boulder, Arapahoe, Adams, Jefferson and El Paso with high population density. Given that the 2013 flooding impacted the Front Range area (Denver MSA, Boulder and Larimer County), and the towns of Jamestown and Lyons (Boulder County) and Estes Park (Larimer County) were isolated by water, it is not surprising that the tweets pertaining to the event, and those containing negative and positive emotions are concentrated in these heavily impacted areas.

Among the machine-learning algorithms examined, SMO performed the best with a correct percentage of $76 \%$ in category prediction and $68.7 \%$ in class prediction (table1). The classifier was acceptable in labeling most classes identified under the category "Information", which included "Warning", "Location" and "Loss" except "Sharing" probably due to its diverse nature (which included embedded links and multimedia). Among "Conversation" category, the classifier was more confused in classifying "Humor" and "Neutral" classes. Due to the ironic and sarcastic nature, the former meme was often filled with polarized context-specific keywords; whereas the "Neutral" class suffered from its vague and broad nature. Due to the small sample sizes in training data for "Preparation", "Positive" and "Help" classes, the classifier performed poorly in classifying these tweets.

\section{CONCLUSION}

The analyses of the tweets indicated that prior to the event a large proportion of tweets were not relevant to the event. During and following the event, the proportion of tweets providing warning, preparatory information, risk information pertaining to loss and damages, and location information increased. The tweets relevant to the event are also concentrated in the areas that experienced severe and moderate damages (Figure 2). However, the tweets providing warning information were generated in areas that were not impacted by the event at all, such as Weld County, which is close to Larimer County that experienced severe damages and Estes Park in Larimer County which was waterlocked. It could be assumed that the tweets containing warnings were generated in least impacted 


\begin{tabular}{|l|l|l|l|l|l|l|l|l|}
\hline & Actual & Conversation & Information & $\begin{array}{l}\text { Not } \\
\text { Relevant }\end{array}$ & Others & $\begin{array}{l}\text { TP } \\
\text { Rate }\end{array}$ & $\begin{array}{l}\text { FP } \\
\text { Rate }\end{array}$ & $\begin{array}{l}\text { ROC } \\
\text { Area }\end{array}$ \\
\hline \multirow{2}{*}{$4^{*}$ Predicted } & Conversation & 336 & 63 & 86 & 0 & 0.711 & 0.122 & 0.834 \\
\cline { 2 - 9 } & Information & 96 & 798 & 94 & 0 & 0.808 & 0.114 & 0.874 \\
\cline { 2 - 9 } & Not Relevant & 128 & 92 & 659 & 2 & 0.748 & 0.12 & 0.871 \\
\cline { 2 - 9 } & Others & 7 & 8 & 4 & 13 & 0.406 & 0.001 & 0.715 \\
\hline
\end{tabular}

Table 1: Summary statistics of categorical classification accuracy

counties as a precautionary measure to alert residents of the event, and help them take appropriate actions (such as evacuate) if needed. The tweets containing location information were concentrated in areas that experienced significant damages. However, these tweets did not provide any specific information about shelters, roads and bridges that were damaged or underwater rather they contained second-hand information that could be used for search and rescue operations instead of response efforts. The Front Range area also had higher concentration of non-relevant tweets, which contained loss and damage information as well as positive and negative sentiments.

The findings suggest that the tweet volume increased in areas that were either significantly impacted by the flooding event or were in close proximity to the areas that were at high risk of being impacted by the event. The temporal distribution of tweet volume also matched the days when the flooding was severe. Because of the low volume of geo-tagged tweets, which is generally the case, and the spatial distribution of these tweets in impacted counties, the SA information derived from these tweets should be combined with physical risk, social vulnerability and extent of damage to geo-target EM efforts. Next phase of the study will focus on (i) analyzing emojis and URLs to extract SA information, (ii) combining SA information extracted from emojis and contents, (iii) assessing physical risk and social vulnerability of the impacted counties, and (iv) relating risk and vulnerability with SA obtained from the geo-tagged tweets. Further analyses will be conducted to explore the spatio-temporal distribution of geo-tagged tweets and the type of SA information available from these tweets with regard to physical risk and social vulnerability of the flood impacted and surrounding counties. This will help determine resilience of impacted communities based on citizen participation and information available to help with EM efforts, and geo-target counties with less citizen participation for enhanced response efforts.

\section{ACKNOWLEDGMENTS}

Bandana Kar has participated in this project in her own independent capacity and not on behalf of UT-Battelle, LLC, or its affiliates or successors. Any opinions, findings and conclusions or recommendations expressed in this manuscript are those of the authors, and do not necessaily reflect the views of DOE or UT-Batelle, LLC.

\section{REFERENCES}

[1] P. Earle, M. Guy, R. Buckmaster, C. Ostrum, S. Horvath, and A. Vaughan. 2010 OMG Earthquake! Can Twitter Improve Earthquake Response? Seismological Research Letters 81, 2 (2010), 246-251.

[2] M. Imran and C. Castillo. 2015. Towards a Data-Driven Approach to Identify Crisis-Related Topics in Social Media Streams. In Proceedings of the 24th International Conference on World Wide Web. 1205-1210.

[3] A. Java, X. Song, T. Finin, and B. Tseng. 2007. Why We Twitter: Understanding Microblogging Usage and Communities. In Proceedings of the foint 9th WEBKDD and 1st SNA-KDD Workshop. San fose, CA. 56-65. https://doi.org/10.1145/1348549. 1348556

[4] N. Kanhabua and W. Nejdl. 2013. Understanding the Diversity of Tweets in the time of Outbreaks. In WWW 2013 Companion.

[5] J. D. Kent and H. T. Capello. 2013. Spatial Patterns and Demographic Indicators of Effective Social Media Content During the Horsethief Canyon fire of 2012. Cartography and Geographic Information Science 40, 2 (2013), 78-89.

[6] A. Kongthon, C. Haruechaiyasak, J. Pailai, and S. Kongyoung. 2014. The Role of Twitter During a Natural Disaster: Case study of 2011 Thai Flood. International fournal of Innovation and Technology Management 11, 3 (2014). https://doi.org/ 10.1142/S0219877014400124

[7] Y. Kryvasheyeu, H. Chen, N. Obradovich, E. Moro, P. Van Hentenryck, J. Fowler, and M. Cebrian. 2016. Rapid Assessment of Disaster Damage using Social Media Activity. Sci. Adv. (2016).

[8] P. M. Landwehr and K. M. Carley. 2014. Social Media in Disaster Relief. In Data Mining and Knowledge Discovery for Big Data, W. W. Chu (Ed.). Springer, Berlin, 225-257.

[9] X. Liu, B. Kar, C. Zhang, and D. Cochran. 2018. Evaluating Relevance of Twitter Data for Risk Communication. International Journal of Digital Earth (2018). https://doi.org/10.1080/17538947.2018.1480670

[10] R. Munro and C. D. Manning. 2012. Short Message Communications: Users, Topics, and In-language Processing. In Proceedings of the 2nd ACM Symposium on Computing for Development. https://doi.org/10.1145/2160601.2160607

[11] A. Olteanu, C. Castillo, F. Diaz, and S. Vieweg. 2014. CrisisLex: A Lexicon for Collecting and Filtering Microblogged Communications in Crises. In Proceedings of ICWSM.

[12] A. Olteanu, S. Vieweg, and C. Castillo. 2015. What to Expect When the Unexpected Happens: Social Media Communications Across Crises. In Proceedings of the 18th ACM Conference on Computer Supported Cooperative Work and Social Computing. 994-1009. https://doi.org/10.1145/2675133.2675242

[13] F. Shaw, J. Burgess, K. Crawford, and A. Bruns. 2013. Sharing News, Making Sense, Saying Thanks: Patterns of Talk on Twitter during the Queensland Floods. Australian fournal of Communication 40, 1 (2013), 23-40. https://doi.org/10.1142/ S0219877014400124

[14] B. Takahashi, E. C. Tandoc Jr., and C. Carmichael. 2015. Communicating on Twitter during A Disaster: An Analysis of Tweets during Typhoon Haiyan in the Philippines. Computers in Human Behavior 50 (2015), 392-398.

[15] I. P. Temnikova, C. Castillo, and S. Vieweg. 2015. EMTerms 1.0: A Terminological Resource for Crisis Tweets. In Proceedings of the International Conference on Information Systems for Crisis Response and Management.

[16] S. Vieweg, A. L. Hughes, K. Starbird, and L. Palen. 2010. Microblogging during Two Natural Hazard Events: What Twitter may Contribute to Situational Awareness. In Proceedings of the SIGCHI conference on Human Factors in Computing Systems. 1079-1088.

[17] B. Wang and J. Zhuang. 2017. Crisis Information Distribution on Twitter: A Content Analysis of Tweets during Hurricane Sandy. Natural Hazards 89, 1 (2017), 161-181. https://doi.org/10.1142/S0219877014400124

[18] H. Wang, E. Hovy, and M. Dredze. 2015. The hurricane Sandy Twitter Corpus. In Association for the Advancement of Artificial Intelligence. 20-24.

[19] S. Zhang and S. Vucetic. 2016. Semi-supervised Discovery of Informative Tweets During the Emerging Disasters. In 4th International Workshop on SWDM, colocated with CIKM. 